\title{
Time correlation function in systems with two coexisting biological species
}

\author{
E. Arashiro, A. L. Rodrigues, M. J. de Oliveira, and T. Tomé* \\ Instituto de Física, Universidade de São Paulo, Caixa Postal 66318, 05315-970 São Paulo, São Paulo, Brazil
}

(Received 24 March 2008; revised manuscript received 19 April 2008; published 12 June 2008)

\begin{abstract}
We study a stochastic lattice model describing the dynamics of coexistence of two interacting biological species. The model comprehends the local processes of birth, death, and diffusion of individuals of each species and is grounded on interaction of the predator-prey type. The species coexistence can be of two types: With self-sustained coupled time oscillations of population densities and without oscillations. We perform numerical simulations of the model on a square lattice and analyze the temporal behavior of each species by computing the time correlation functions as well as the spectral densities. This analysis provides an appropriate characterization of the different types of coexistence. It is also used to examine linked population cycles in nature and in experiment.
\end{abstract}

DOI: 10.1103/PhysRevE.77.061909

PACS number(s): 87.23.Cc, 87.10.Hk, 05.70.Ln

\section{INTRODUCTION}

The Lotka-Volterra model is the first one [1,2] describing how the interaction between two biological species leads to time oscillations. This model consists of a set of two coupled ordinary differential equations for the density of prey and predator and is set up in analogy with the laws of mass action [3-6]. However, the Lotka-Volterra description does not take in an explicit way the spatial structure of the environment where the species coexist. One manner of taking into account the spatial structure is to consider lattice models in which the species individuals reside on the sites of the lattice and interact with its neighbors by stochastic local transition rules [7-13,15-27,29-31]. These models are usually continuous time Markovian processes defined by discrete stochastic variables residing on the sites of the lattice, also called interacting particle system $[32,33]$.

The stochastic transition rules embodied in the lattice model imply stochastic fluctuations in the number of a given species as a function of time. The stochasticity, which is ubiquitous in nature and is observed in real data, is also an essential ingredient of the stochastic lattice models, object of our analysis, but is absent in the Lotka-Volterra model. The stochastic lattice models, moreover, give a correct description of the local time oscillations in the sense that the amplitude of the oscillations do not depend on the initial conditions. The oscillations coming from the stochastic lattice models are autonomous, or self-sustained, and depend only on the external parameter. The stochastic lattice model as well as some other approaches [34-38], incorporates the discreteness of the species individuals and local stochastic interactions which are important ingredients to describe cyclic fluctuation in population dynamics.

In the present work we study the coexistence and the emergence of stable local self-sustained oscillations in a stochastic lattice model that describes the interaction between two species, in particular, predator and prey species. Here we study a modified version of the model introduced by Satulovsky and Tomé [8] by including diffusion of species indi-

\footnotetext{
*Corresponding author; ttome@if.usp.br
}

viduals. It has been pointed out that diffusion provides a more realistic description since the species individuals move themselves in their habitat [27,39-45]. We focus on the analysis of the time series by determining the time autocorrelation function of each species as well as the time crosscorrelation function. As applications of this type of analysis we examine two examples of real data coming from coexisting biological species found in nature and in experiments.

\section{MODEL}

We depart from the stochastic lattice model for a predatorprey system [8]. One considers a regular square lattice representing the habitat where the two interacting species coexist. Each site can be either empty or occupied by one individual of different species. The state of a site is described by a variable $\eta_{i}$ that takes the values 0,1 , or 2 , according to whether the site is empty, occupied by a prey individual or by a predator, respectively. That is,

$$
\eta_{i}=\left\{\begin{array}{lc}
0, & \text { empty, } \\
1, & \text { prey }, \\
2, & \text { predator } .
\end{array}\right.
$$

A microscopic state of the system is denoted by the stochastic vector $\eta=\left(\eta_{1}, \ldots, \eta_{i}, \ldots, \eta_{N}\right)$, where $N$ is the total number of sites. The probability $P(\eta, t)$ of configuration $\eta$ at time $t$ evolves in time according to the master equation

$$
\frac{d}{d t} P(\eta, t)=\sum_{\eta^{\prime}}\left\{W\left(\eta, \eta^{\prime}\right) P\left(\eta^{\prime}, t\right)-W\left(\eta^{\prime}, \eta\right) P(\eta, t)\right\},
$$

where the summation is over all the microscopic configurations $\eta^{\prime}(\neq \eta)$ of the system, and $W\left(\eta, \eta^{\prime}\right)$ is the rate of transition (conditional probability per unit time) from state $\eta^{\prime}$ to state $\eta$.

The transitions between the three states obey the cyclic order shown in Fig. 1. The three processes are described as follows: Prey individuals are born in empty sites; prey individual dies and is instantaneously replaced by a new born predator; finally a predator can die leaving an empty site. The two first processes are catalytic whereas the third is spontaneous. 


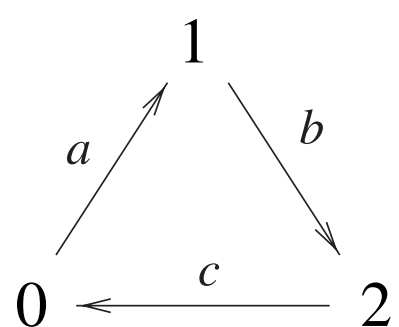

FIG. 1. Transitions of the predator-prey model. The three states are prey (1), predator (2), and empty (0). The allowed transitions obey the cyclic order shown.

The predator-prey lattice model has three parameters: $a$, the probability of birth of prey, $b$, the probability of birth of predator and death of prey, and $c$, the probability of predator death. The occupancy of a site by prey or by a predator is conditioned, respectively, to the existence of prey or predator in the neighborhood of the site. The third reaction, where predator dies occurs with probability $c$, independently of the states of the neighboring sites. By rescaling time it is possible to assume that $a+b+c=1$, with $0 \leq a, b, c \leq 1$. We use an auxiliary parameter $p$ and write $a=(1-c) / 2-p$ and $b$ $=(1-c) / 2+p$.

More specifically, the transition probabilities are described in what follows:

(a) If a site $i$ is empty $\left(\eta_{i}=0\right)$ and there is at least one prey individual in its first neighborhood, there is a favorable condition for the birth of a new prey. The probability of site $i$ being occupied in the next time step by a prey is proportional to the parameter $a$ and to the number of prey individuals in the first neighborhood of the empty site. The transition probability per site associated to this process is given by

$$
w_{i}(0 \rightarrow 1)=\delta\left(\eta_{i}, 0\right) \frac{a}{4} \sum_{k} \delta\left(\eta_{k}, 1\right),
$$

where the summation is over the four nearest neighbors of site $i$ in a regular square lattice. The notation $\delta(x, y)$ stands for the Kronecker $\delta$ function.

(b) If a site is occupied by a prey $\left(\eta_{i}=1\right)$ and there is at least one predator in its first neighborhood, then the site has a probability of being occupied by a new predator in the next time step. In this process the prey dies instantaneously. The transition probability is proportional to the parameter $b$ and to the number of predators in the first neighborhood of the site. The transition probability for this process is

$$
w_{i}(1 \rightarrow 2)=\delta\left(\eta_{i}, 1\right) \frac{b}{4} \sum_{k} \delta\left(\eta_{k}, 2\right) .
$$

(c) If site $i$ is occupied by a predator $\left(\eta_{i}=2\right)$ it dies with probability $c$. The corresponding transition probability is given by

$$
w_{i}(2 \rightarrow 0)=c \delta\left(\eta_{i}, 2\right),
$$

These stochastic local rules define the dynamics of the stochastic lattice model for a predator-prey system without diffusion.

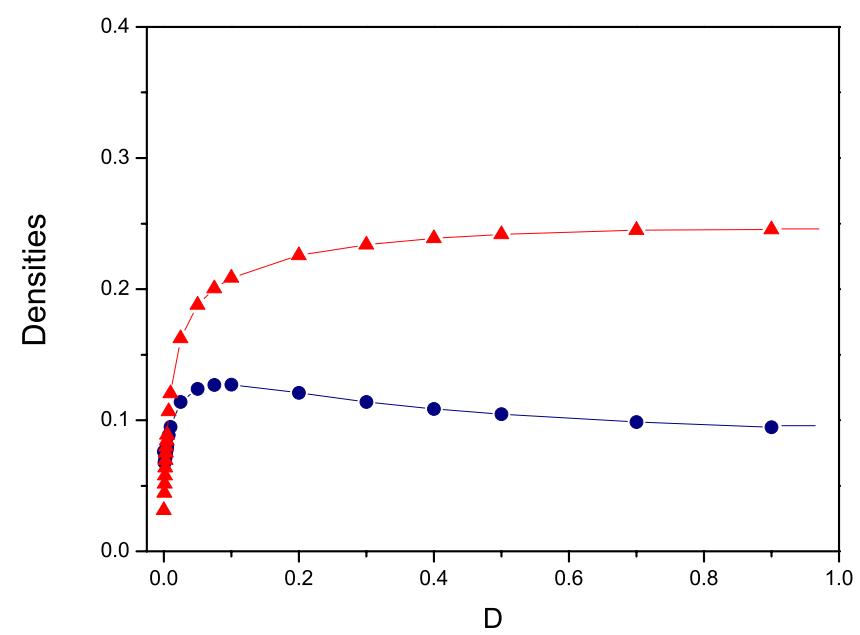

FIG. 2. (Color online) Average densities of prey (lower points) and predator (upper points) for $p=0.3$ and $c=0.05$ as a function of D.

Here we consider a reaction-diffusion process by adding to the above transitions, related to the reactive processes, a diffusion process to mimic the explicit movement of prey. That is, with probability $D$ a diffusion is attempted; with probability $1-D$ one performs the transitions (a), (b), and (c) above. The diffusion is realized as follows. One site is chosen at random. Suppose it is occupied by one prey individual, there are $n$ empty nearest-neighbor sites. Then the individual jumps to one of the $n$ empty sites with equal probability. In analogous way, if the chosen site is empty and there are $n$ nearest-neighbor prey individuals, one of them is chosen at random and jumps to the empty site. Otherwise, that is, if the chosen site is occupied by a predator state of sites remains unchanged.

\section{TIME CORRELATION}

To characterize the time behavior of the density of the two species, we measure the time autocorrelation function for each species and the time cross-correlation function between the two species. Let us denote by $x(t)$ and $y(t)$ the times series for the prey and predator, respectively, and let $\bar{x}$ and $\bar{y}$ be their respective average in time. We define the prey and predator time autocorrelation functions as

$$
c_{x}(t)=\int\left[x\left(t^{\prime}+t\right)-\bar{x}\right]\left[x\left(t^{\prime}\right)-\bar{x}\right] d t^{\prime}
$$

and

$$
c_{y}(t)=\int\left[y\left(t^{\prime}+t\right)-\bar{y}\right]\left[y\left(t^{\prime}\right)-\bar{y}\right] d t^{\prime} .
$$

The time cross-correlation function between prey and predator is given by

$$
c_{x y}(t)=\int\left[x\left(t^{\prime}+t\right)-\bar{x}\right]\left[y\left(t^{\prime}\right)-\bar{y}\right] d t^{\prime} .
$$

Instead of these correlations we use in actual calculations the normalized correlations, defined by 

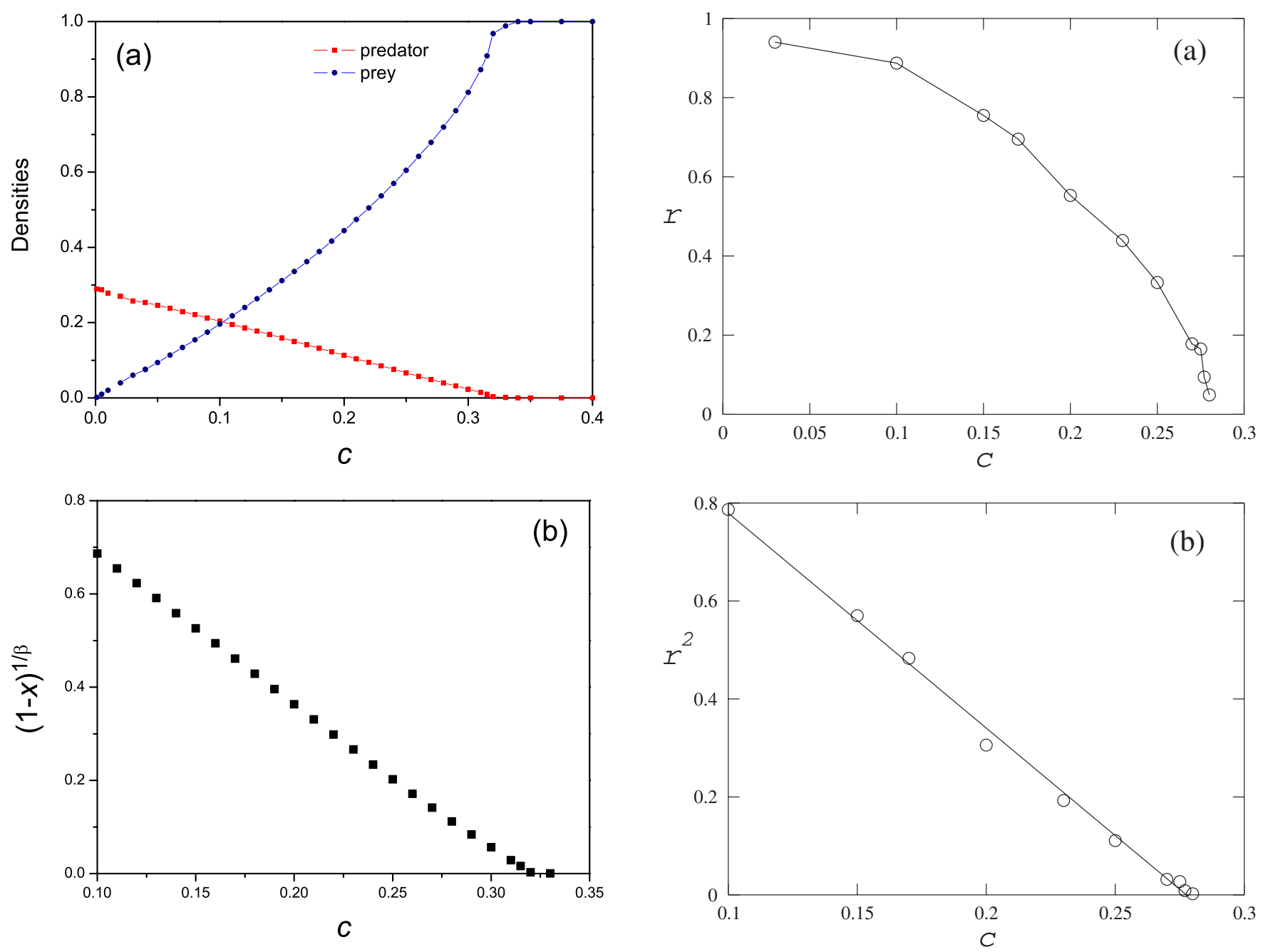

FIG. 3. (Color online) (a) Density of prey $x$ and density of predator $y$ versus $c$ for $p=0.3$ and $D=0.9$. (b) The quantity (1 $-x)^{1 / \beta}$ versus $c$, where $\beta=0.58$. The critical point occurs at $c_{c}$ $=0.320(5)$.

$$
\begin{aligned}
& r_{x}(t)=\frac{c_{x}(t)}{c_{x}(0)}, \\
& r_{y}(t)=\frac{c_{y}(t)}{c_{y}(0)}, \\
& r_{x y}(t)=\frac{c_{x y}(t)}{c_{x y}(0)} .
\end{aligned}
$$

For each of the two autocorrelation functions, we have determined the Fourier transform, given by

$$
\begin{aligned}
& S_{x}(\omega)=\int r_{x}(t) e^{i \omega t} d t, \\
& S_{y}(\omega)=\int r_{y}(t) e^{i \omega t} d t .
\end{aligned}
$$

According to the Wiener-Kinchin theorem, $S_{x}(\omega)$ and $S_{y}(\omega)$ may be identified with the power spectral density relative to prey and predator time series, respectively.

FIG. 4. (a) Value of the cross-correlation $r$ at the first maximum as a function of the parameter $c$, for $p=0.3$ and $D=0.9$. (b) Plot of $r^{2}$ as functions of $c$. The linear regression gives the value $c^{*}$ $=0.278(5)$ for the transition from oscillatory to ordinary coexistence.

Let us assume that the correlation function is described, at least qualitatively, by the function

$$
r(t)=e^{-\gamma|t|} f(t)
$$

where $\gamma$ is a parameter describing the time decay and $f(t)$ is a periodic even function of time with a certain period. If we expand $f(t)$ in Fourier series we obtain

$$
r(t)=e^{-\gamma|t|} \sum_{n} A_{n} \cos \left(n \omega_{0} t\right),
$$

where $A_{n}$ are the amplitudes and $\omega_{0}$ is the main frequency.

The Fourier transform of the correlation $r(t)$ is then

$$
S(\omega)=\sum_{n} A_{n}\left(\frac{\gamma}{\gamma^{2}+\left(n \omega_{0}+\omega\right)^{2}}+\frac{\gamma}{\gamma^{2}+\left(n \omega_{0}-\omega\right)^{2}}\right),
$$

which has local maxima at $\omega=n \omega_{0}$. For large values of $\omega$, that is, $\omega \gg \gamma$, and assuming that $A_{n}$ decreases rapidly with $n$, we have 

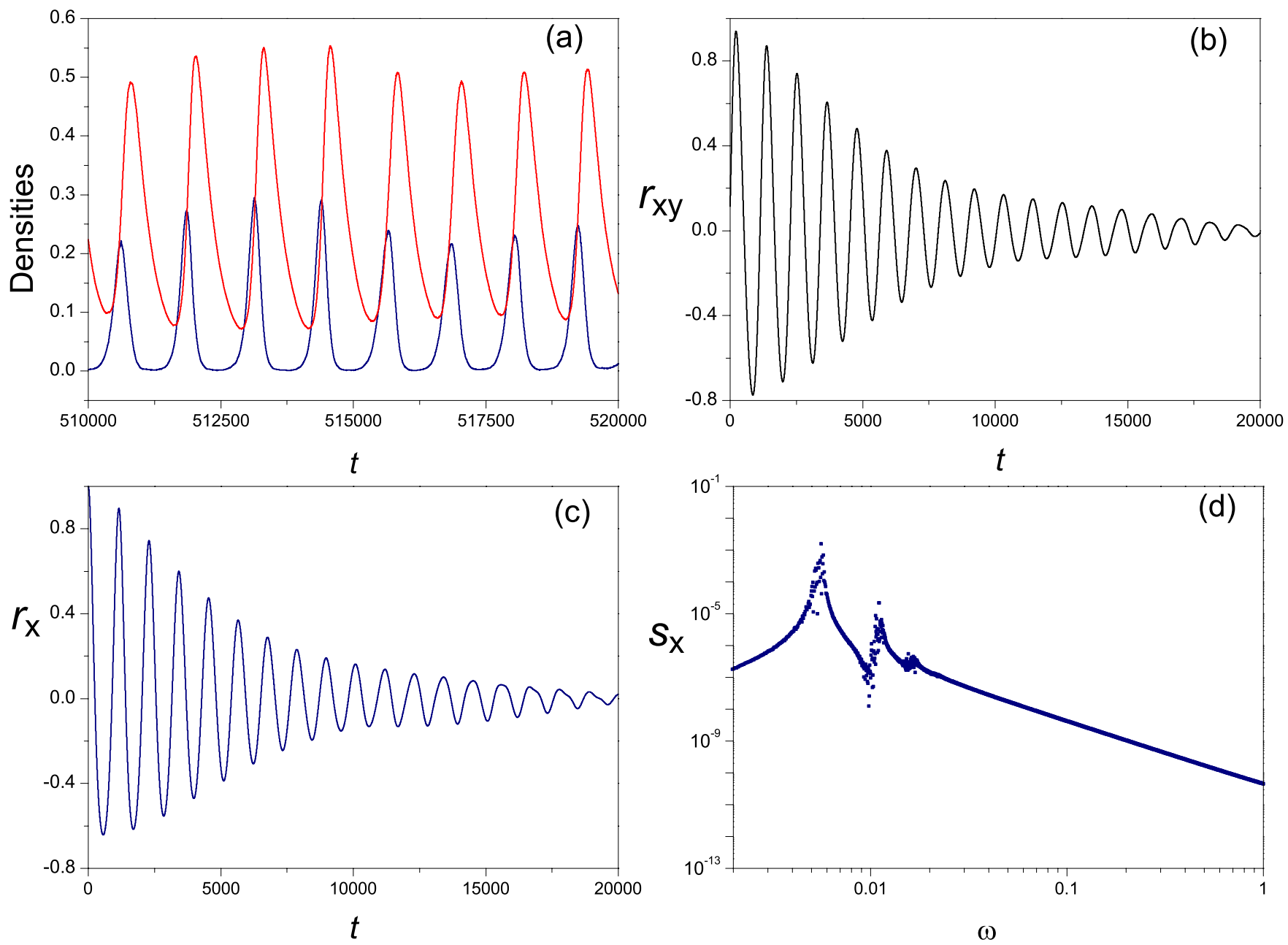

FIG. 5. (Color online) (a) Densities of prey (lower curve) and predator (upper curve) as a function of time for $p=0.3, c=0.03$, and $D$ =0.9. (b) Cross-correlation function between prey and predator. (c) Time autocorrelation function for prey. (d) Power spectral density for prey. The largest peak occurs at $\omega=0.0057$ which gives a period $T=1.10 \times 10^{3}$. The second and the third peaks occur at $\omega=0.0112$ and $\omega=0.016$. The slope of the tail equals $\alpha=2.0$.

$$
S(\omega) \sim \frac{\gamma}{\omega^{\alpha}}, \quad \alpha=2
$$

so that the $\log -\log$ plot of $S$ versus $\omega$ has a slope $\alpha=2$ characterizing the exponential decay of the correlation function.

In the absence of oscillation we may describe the time autocorrelation function qualitatively by the following function:

$$
r(t)=A_{0} e^{-\gamma|t|}
$$

which may be understood as the expression (15) in which all coefficients $A_{n}$ vanish except the coefficient $A_{0}$. Its Fourier transform is given by the Lorentzian

$$
S(\omega)=\frac{2 A_{0} \gamma}{\gamma^{2}+\omega^{2}},
$$

and is a monotonic decreasing function of frequency. For large values of $\omega$, that is, $\omega \gg \gamma$, the Fourier transform behaves according to Eq. (17).

\section{NUMERICAL SIMULATION}

The simulations of the stochastic lattice model were performed in a square lattice of $N=160 \times 160$ sites starting from a random configuration of prey and predator in which each site has a probability $1 / 3$ of being occupied by a predator and $1 / 3$ of being occupied by a prey individual. The total number of Monte Carlo steps ranged from $10^{5}$ to $10^{6}$. At each time step the configuration of the lattice was updated according to the stochastic rules presented in Sec. II. To explore the several possible states of the model we may use a phase diagram in the variables $p$ and $c$ for a given fixed value of the diffusion parameter $D$. Since we are concerned with the characterization of the time series, it suffices to consider a representative section of the phase diagram, which we choose to be $p=0.3$ and $D=0.9$. The choice of $D$ is discussed in what follows.

The explicit diffusion enhances the species coexistence and the oscillatory behavior. The introduction of the diffusion process promotes the increase of the average species densities as can be seen in Fig. 2. Even a small value of $D$ suffices to entail this behavior as shown by the rapid increase 

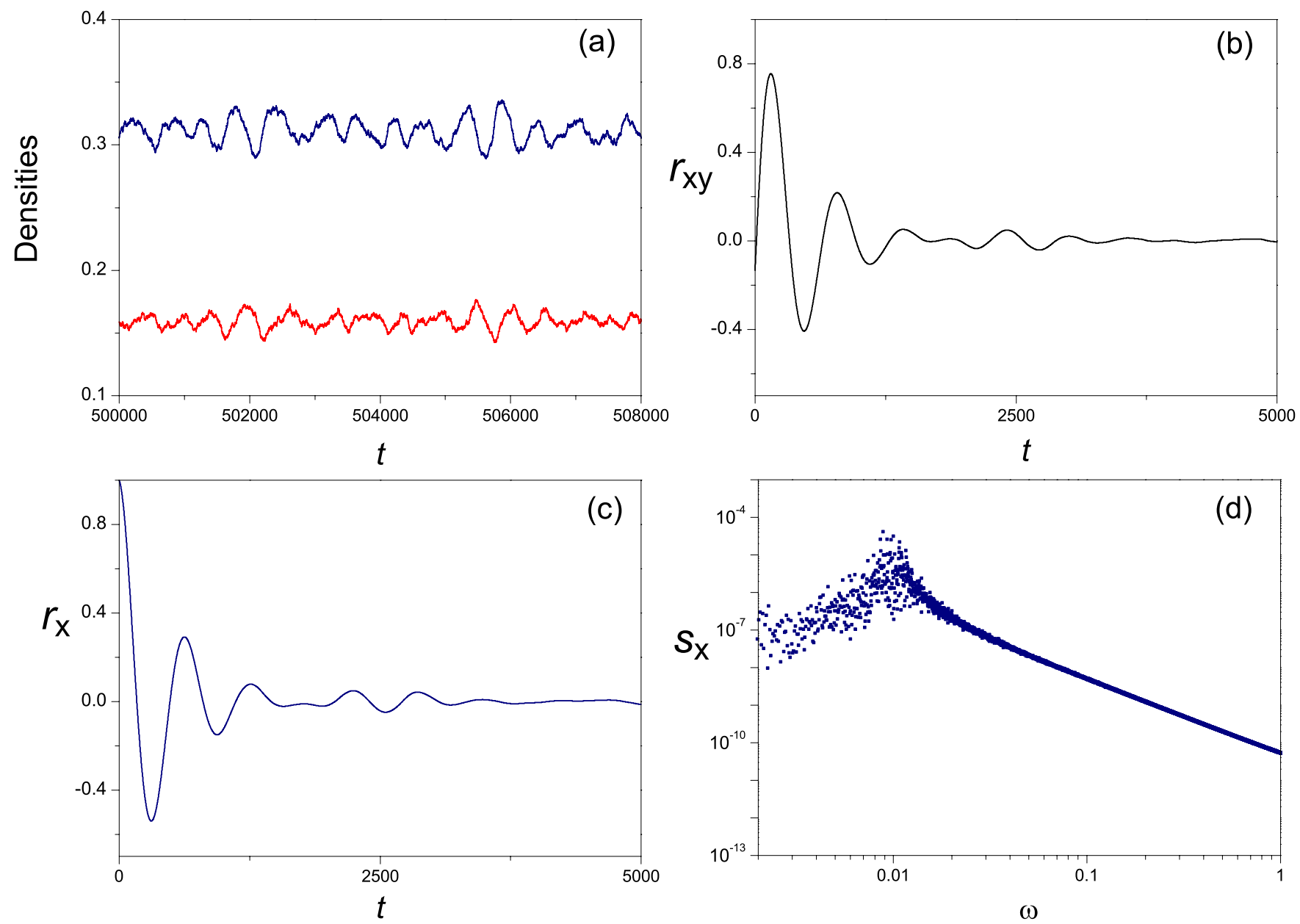

FIG. 6. (Color online) (a) Densities of prey (upper curve) and predator (lower curve) as a function of time for $p=0.3, c=0.15$, and $D$ =0.9. (b) Cross-correlation function between prey and predator. (b) Time autocorrelation function for prey. (d) Power spectral density for prey. The largest peak occurs at $\omega=0.0102$ which gives a period $T=616$. The slope of the tail equals $\alpha=2.0$.

in the average species densities up to $D \cong 0.05$. Above this value the average densities become almost constant indicating that the behavior of the system is similar for values of $D>0.05$. It is therefore enough to study the properties of the system for just one point within this interval, which we choose here to be $D=0.9$.

The present model predicts a state in which the lattice is full of prey, called prey absorbing state, in which the system cannot escape, remaining there forever. This absorbing state occurs if the parameter $c$, that regulates the death of predator, is sufficiently high. As one increases the external parameter $c$ the system then shows a continuous phase transition from a state where the species coexist to a prey absorbing state. For $D=0.9$ and $p=0.3$, this occurs at a critical value $c_{c}$ as shown in Fig. 3, where the averages $x$ and $y$, density of prey and predator, respectively, are plotted as a function of $c$. The prey absorbing state is characterized by $x=1$ and $y=0$. In this figure we have also plotted $(1-x)^{1 / \beta}$ as a function of $c$. Since we expect that this critical behavior belongs to the universality class of direct percolation $[25,29]$ we used $\beta=0.58$. The straight line fitted to the data points corroborates this expectation and gives the value $c_{c}=0.320(5)$.

In the interval between $c=0$ and $c=c_{c}$ the system exhibits the most interesting states, which are the active states where prey and predator coexist. We distinguish two types of coexistence: One with time oscillatory behavior, for small values of $c$, and the other without oscillations, which we call ordinary coexistence, for values of $c$ near $c_{c}$. Our numerical study predicts the change from one behavior to another at $c=c^{*} \approx 0.28$ for $p=0.3$ and $D=0.9$, as can be inferred from Fig. 4, and to be explained shortly.

In Figs. 5-8 we show the densities of prey and predator as functions of time for $p=0.3, D=0.9$ and for the following values of $c: 0.03,0.15,0.25$, and 0.29 , respectively. We present also the time autocorrelation function for prey, the cross-correlation between prey and predator, and the Fourier transform of the autocorrelation of prey. For the first three values of $c$ the system shows clear oscillations characterized by oscillating correlation functions whose amplitudes decay exponentially.

The plots in Figs. 5-7, indicate that the correlation and the power spectral density are in qualitative agreement with Eqs. (15) and (16). Moreover, the slope of the tail is equal to $\alpha$ $=2$, in agreement with Eq. (17), confirming the exponential decay of the correlation function. In Fig. 8, corresponding to a value of $c$ nearly above $c^{*}$, where no oscillatory behavior is expected, the plots are in qualitative agreement with Eqs. (18) and (19). The slope of the tail is equal to $\alpha=2$, in agree- 

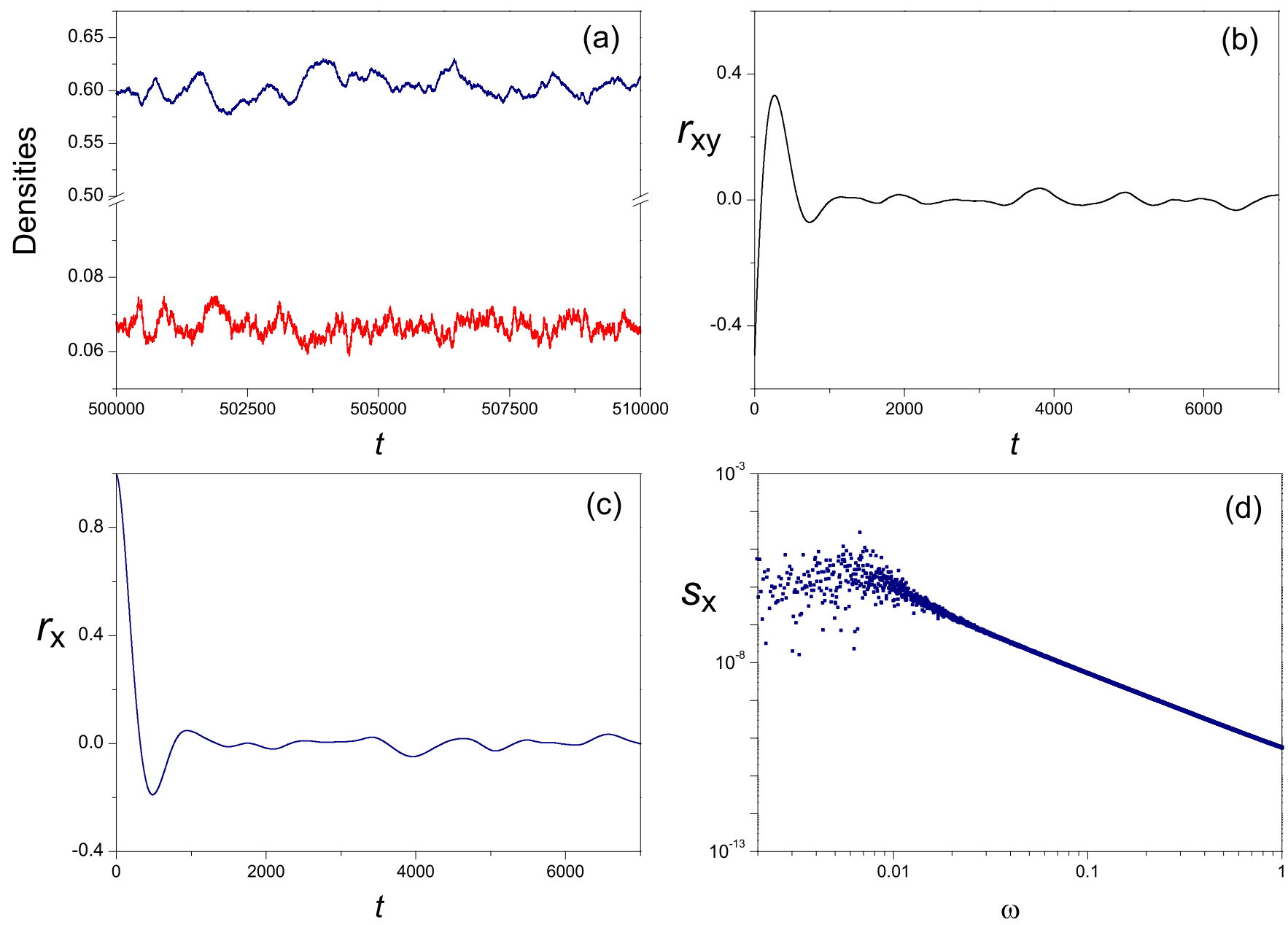

FIG. 7. (Color online) (a) Densities of prey (upper curve) and predator (lower curve) as a function of time for $p=0.3, c=0.25$, and $D$ =0.9. (b) Cross-correlation function between prey and predator. (c) Time autocorrelation function for prey. (d) Power spectral density for prey. The largest peak occurs at $\omega=0.007$ which gives a period $T=9 \times 10^{2}$. The slope of the tail equals $\alpha=2.0$.

ment with Eq. (17), confirming the exponential decay of the correlation function, characterizing the fluctuations as Brownian noise.

We observe that the time behavior of the cross-correlation function between the predator and prey shown in Figs. 5-8 are very similar to the respective autocorrelation functions. For the cases of Figs. 5-7, corresponding to the oscillatory coexistence, apart from a phase shift, the cross-correlation behaves just like the autocorrelation. We notice also that the first maximum of $r_{x y}$ occurs at a nonzero value of the time lag implying that the predator oscillations are delayed with respect to the prey oscillations and that a maximum in prey population is followed by a maximum in predator population. For instance, in Fig. 6 we can observe that the first maximum of $r_{x y}$ as a function of time occurs at approximately one-quarter cycle.

The oscillations are not observed at a global level, that is, they are not synchronized oscillations. However, they can be understood as oscillations occurring at a local level in the sense that the amplitudes of the oscillations in predator-prey lattice models, in two-dimensions and with no diffusion, decreases as $1 / \sqrt{N}$ as the size $N$ of the system increases $[8,14,19,26,28]$. This result is also observed in a class of predator-prey lattice models with explicit diffusion $[14,26,28]$, such as the one studied here as can be seen in Fig. 9, where the oscillations in prey densities are shown for different system sizes. The plot of the amplitude in density $A$ versus the system size $N$ shows that indeed $A$ decreases as $1 / \sqrt{N}$. Therefore, although the explicit diffusion enhances the oscillatory behavior it is not sufficient to hinder the washing out of the oscillatory behavior in the thermodynamic limit. In spite of the vanishing of $A$, the Fourier transform of the autocorrelation function for prey, that is, the power spectrum density for prey, for distinct system sizes, remains with the same form; the peaks are in the same places, independently of the system size as shown in Fig. 9. This behavior of the power spectrum density was also observed in similar systems [26].

As one increases the parameter $c$ the oscillation decreases and ceases above the value $c^{*}$. Below this value, for instance, at $c=0.03$, the trajectories in the plane $x-y$ form a stochastic limit cycle as shown in Fig. 10. For other values of $c$ below $c^{*}$ the cycles exist but their sizes are smaller than the one for $c=0.03$ and become blurred by fluctuations as can be seen in the same figure. As one approaches the value $c=c^{*}$ the average radius of the limit cycle seems to vanish. To determine $c^{*}$ 

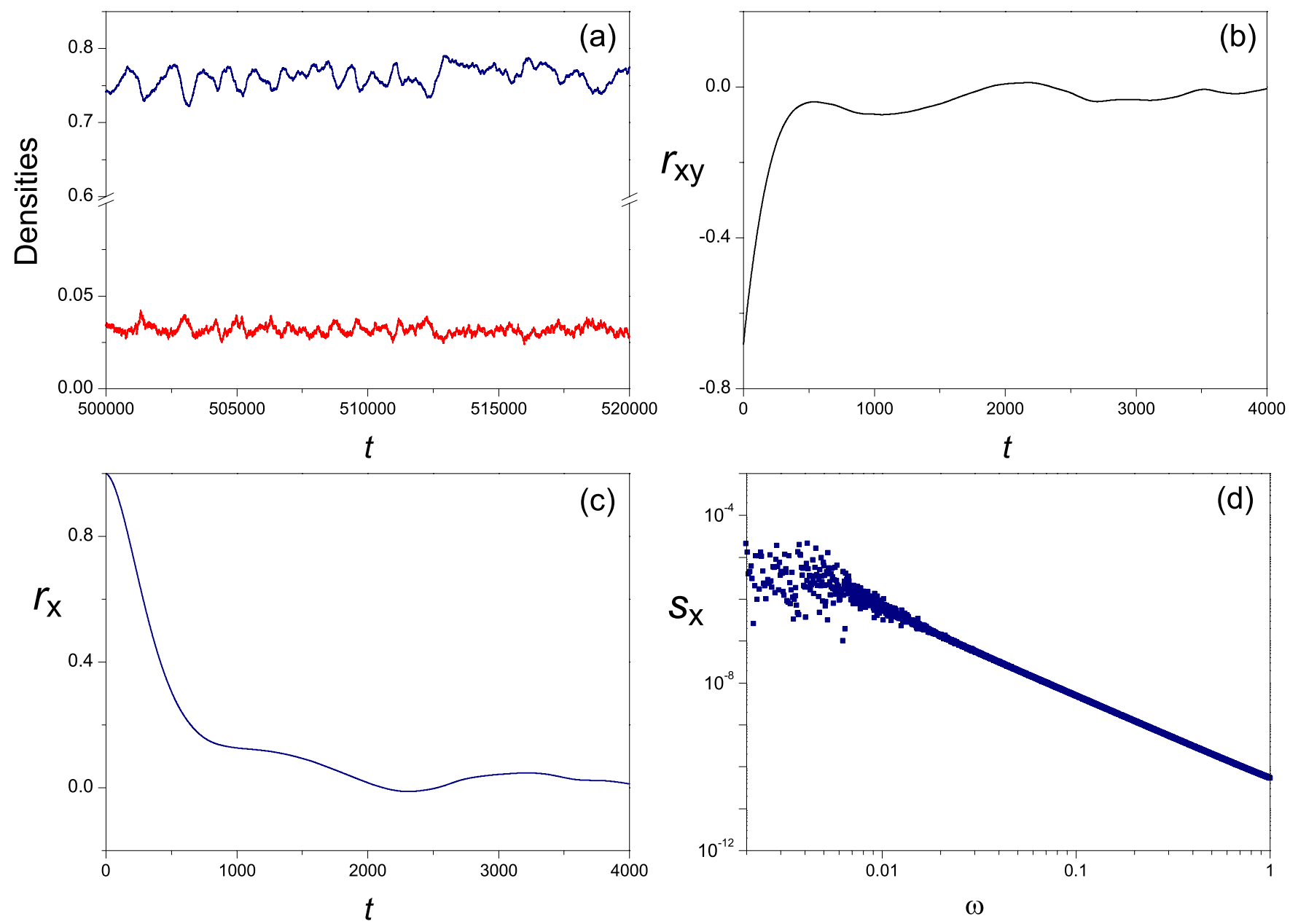

FIG. 8. (Color online) (a) Densities of prey (upper curve) and predator (lower curve) as a function of time for $p=0.3, c=0.29$, and $D$ =0.9. (b) Cross-correlation function between prey and predator. (c) Time autocorrelation function for prey. (d) Power spectral density for prey. The slope of the tail equals $\alpha=2.0$.

we proceed as follows. From the cross-correlation function we determine, for each value of $c$, the values $r_{1}, r_{2}, \ldots$, of the first local maximum, the second local maximum, and so on. The plot of $r_{1}$, for instance, as a function of $c$, as shown in Fig. 4 , indicates that $r_{1}$ vanishes at the $c^{*}$. Near $c=c^{*}, r_{1}$ behaves as $\left(c^{*}-c\right)^{1 / 2}$.

\section{REAL DATA EXAMPLES}

The above-mentioned features related to the oscillating coexistence of the two species, predicted by the stochastic lattice model, namely, the persistence of prey and predator over a large number of periods of time, the lag of predator relative to prey, a peak in prey abundance followed by a peak in predator abundance, and the independence from the initial conditions, are actually observed in real data for predatorprey and host-parasite population cycles as the examples shown in Figs. 11 and 12, respectively.

In Fig. 11 we present data corresponding to the fluctuation in the abundance of snowshoe hare and Canadian lynx in Canada [6]. Using these data we determined the time crosscorrelation function and the time autocorrelation functions for hare and lynx. From these last functions we calculated the respective power spectral densities. One observes that the cross-correlation function implies a lag of lynx relative to hare of about one-quarter of a cycle. The correlation functions show clearly that the species are synchronized with a cycle about 10 years. This period of 10 years is corroborated by a peak in the power spectra for both species. The decay of the power spectra for large $\omega$ is not clearly defined since the tail is very short. However, it seems to be consistent with the behavior given by (17).

As a second example we show in Fig. 12 one of the experimental populations obtained in the laboratory by Utida [46] from a mixed population of the azuki bean weevil, the host, and its parasite larval wasp. From the population densities as functions of time (in generations) we have determined the cross-correlation function and the two autocorrelation functions as well as the respective power spectral density. From the correlation we conclude that the period $T$ $\approx 7$ generations. This result is corroborated by a maximum peak in the power spectra, occurring at $w \approx 0.9$ which gives $T \approx 7$.

Concerning the Canadian lynx and the snowshoe hare data, it is worth noticing that although the cross-correlation function shows that the two populations are indeed coupled, 

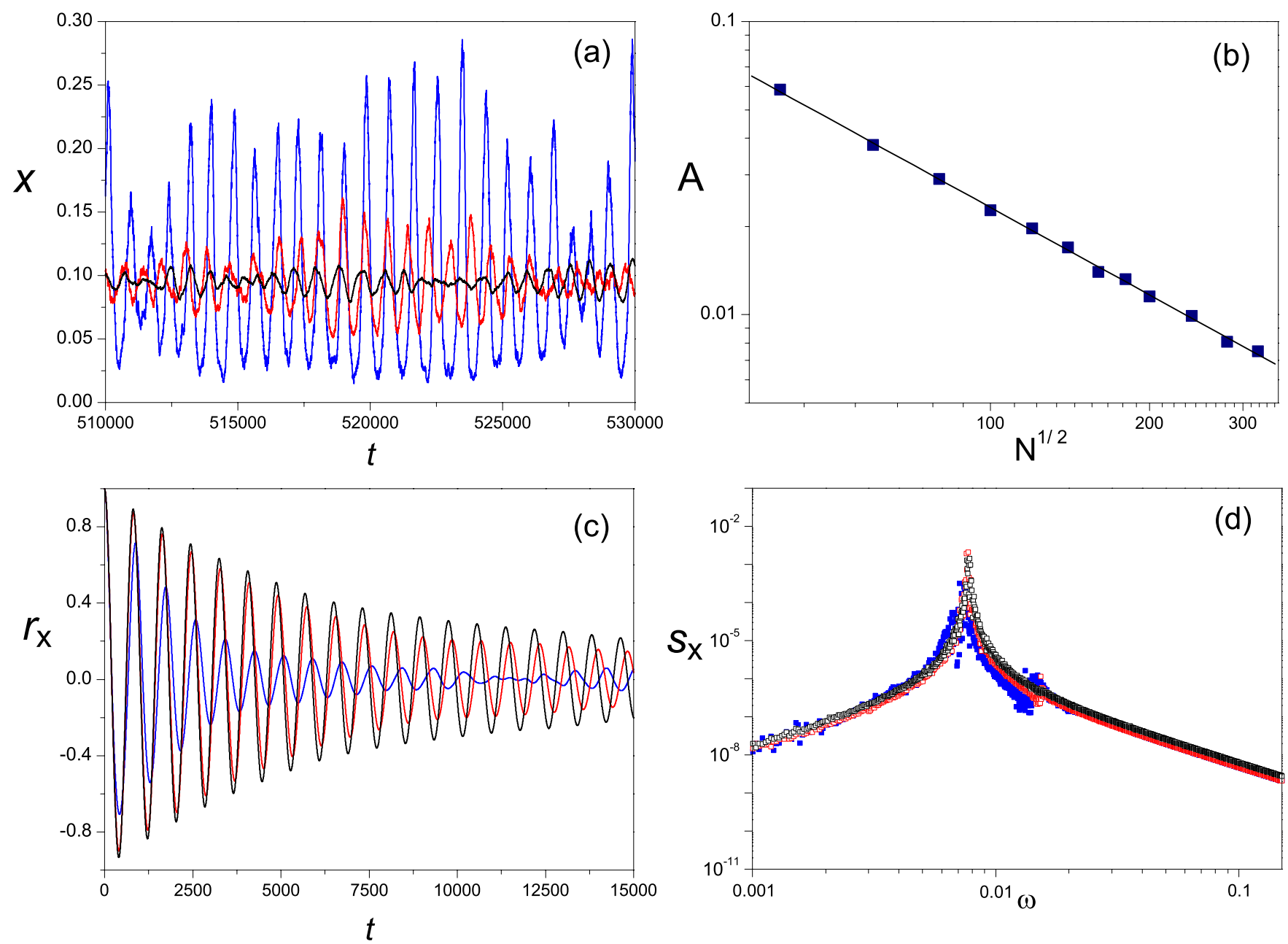

FIG. 9. (Color online) Simulational data obtained at $p=0.3, c=0.05$, and $D=0.9$. (a) Densities of prey as a function of time for $L=40$, 100, and 320, from bigger to smaller amplitudes. (b) Amplitude of prey density oscillations as a function of $\sqrt{N}$, where $N=L \times L$ is the total number of sites. The linear behavior of the $\log -\log$ plot shows that $A \sim 1 / \sqrt{N}$. (c) Time autocorrelation function for prey for $L=40,100$, and 320. (d) Power spectral density for prey for $L=40,100$, and 320.

the cause of the observed correlation between these two populations may not be generated just from their mutual interaction, but may have other causes. It has been pointed out $[47,48]$ that the oscillations in hare appear to be regulated not just by the interaction of the hare with the lynx but also by the hare food supply. The lynx oscillations in turn appear to be induced by the oscillations in the snowshoe hare [47]. On the other hand, the dynamics of the azuki weevil and its parasite can be considered as typical dynamics of the hostparasitoid interaction which in turn is similar to predatorprey interaction [5].

\section{CONCLUSION}

We have studied a stochastic lattice model describing a predator-prey system. By means of numerical simulations of the model defined on a square lattice, the time series of the

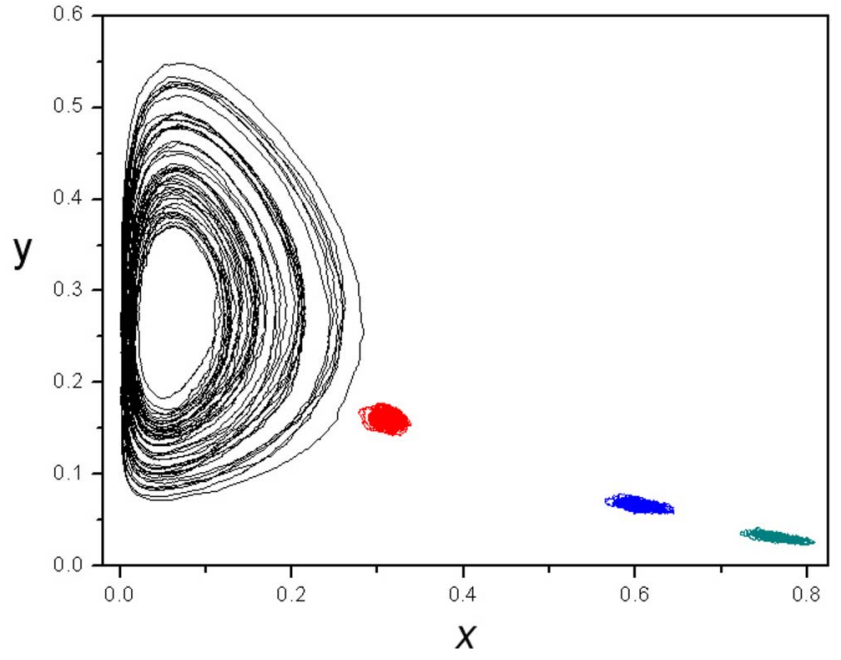

FIG. 10. (Color online) Predator density, $y$, versus prey density, $x$, corresponding to $p=0.3$ and $D=0.9$, for $c=0.03, c=0.15$, $c=0.25$, and $c=0.29$, from the left-hand side to the right-hand side, respectively. 

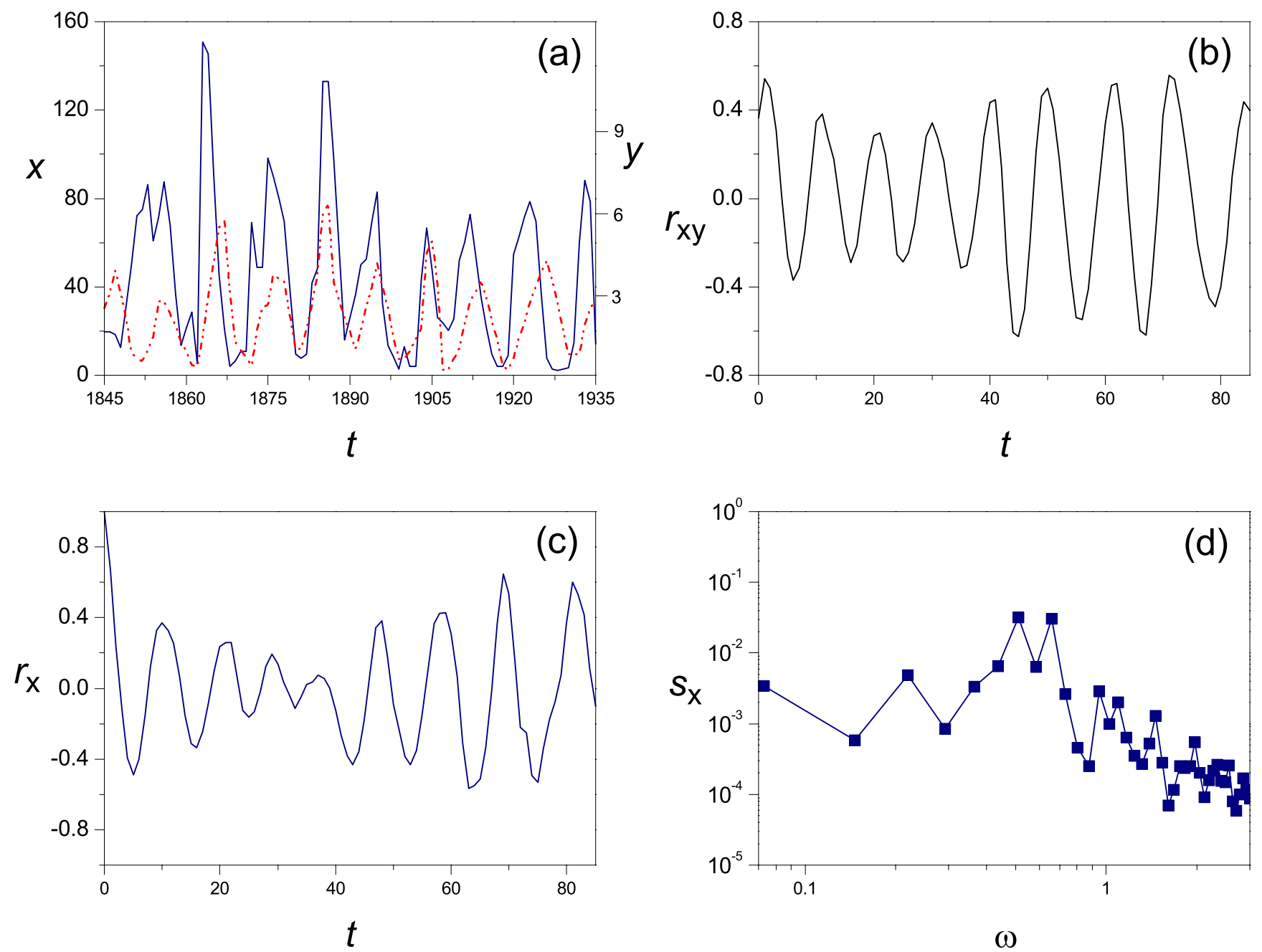

$\omega$
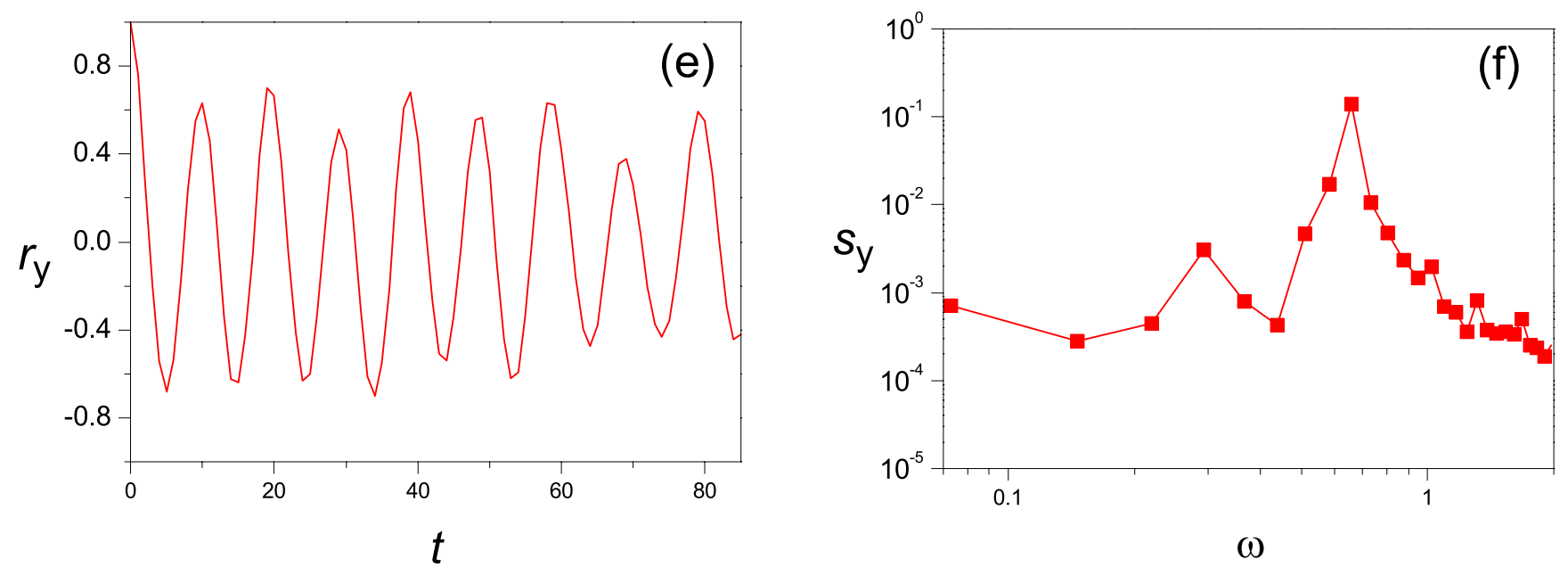

FIG. 11. (Color online) Oscillatory time behavior in the abundance of the snowshoe hare and the Canadian lynx in Canada. (a) Number of lynx ( $y$, dotted curve) and number of hares ( $x$, continuous curve) in thousands as a function of time in years (redrawn from Ref. [6]). (b) Cross-correlation function between lynx and hare. (c) Time autocorrelation function for hare. (d) Power spectral density for hare. (e) Time autocorrelation function for lynx. (f) Power spectral density for lynx.

population densities of each species were obtained. The series were analyzed by determining the time autocorrelation functions for prey and for predator from which we calculate the respective power spectra densities. The time crosscorrelation function between prey and predator was also determined. The results coming from this analysis indicate that 

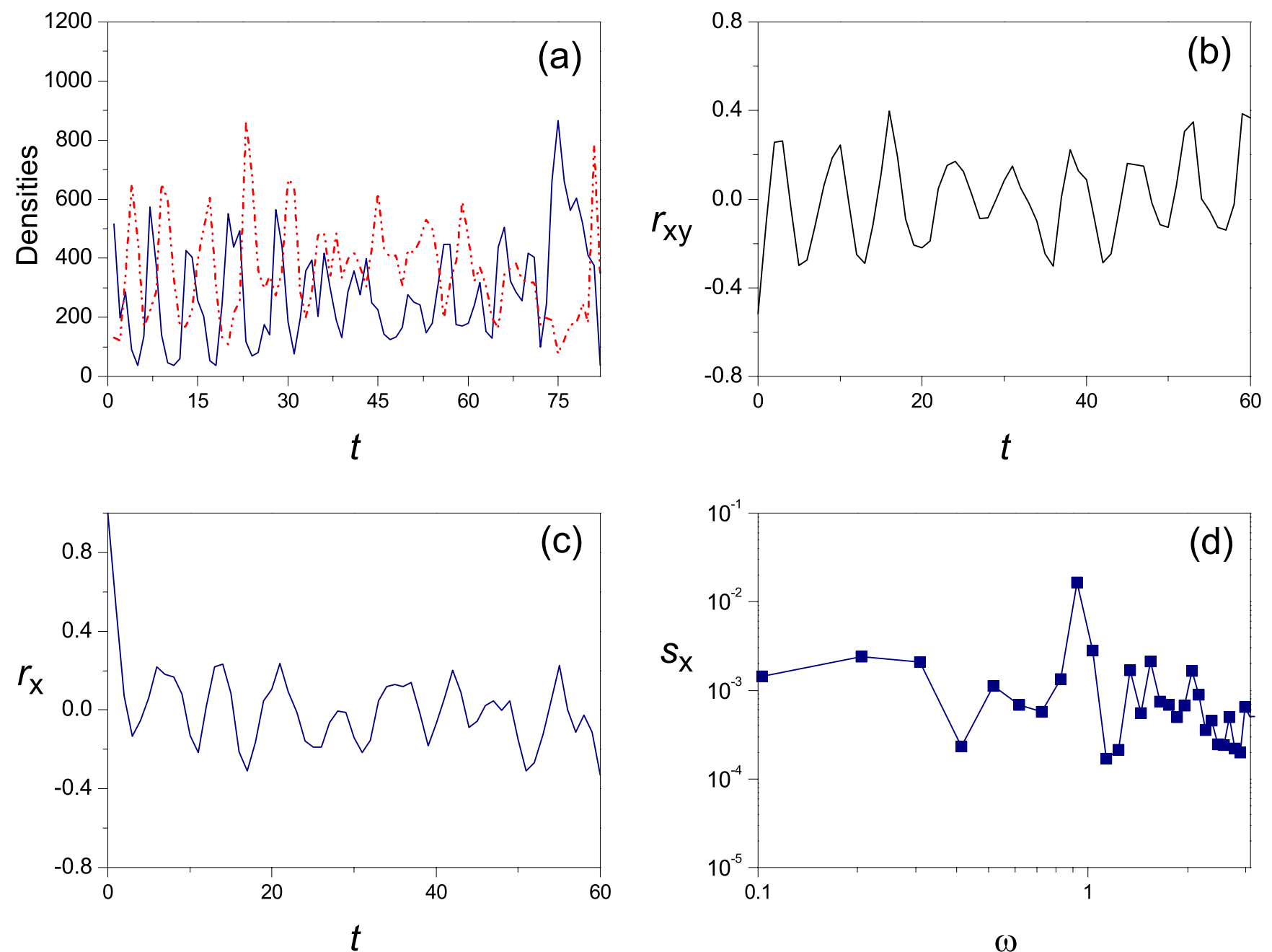

$\omega$
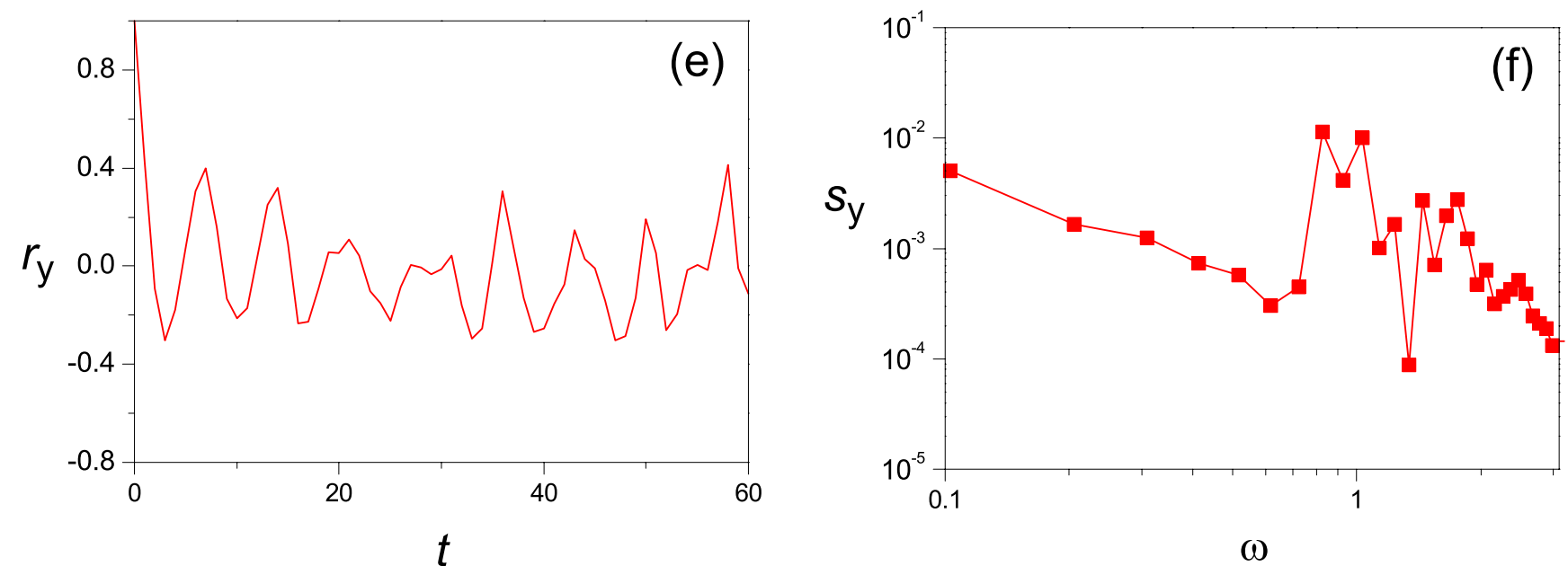

FIG. 12. (Color online) Oscillation in the populations of the host-parasite system azuki weevil and its parasite larval wasp [46]. (a) Population versus time in generations: Parasite population (dotted curve) and host population (continuous curve) (redrawn from Ref. [46]). (b) Cross-correlation function between host and parasite. (c) Autocorrelation for host. (d) Power spectral density of host. (e) Autocorrelation for parasite. (f) Power spectral density of parasite.

the stochastic lattice model is able to predict species coexistence with linked cycles of species populations and ordinary coexistence, that is, coexistence without oscillations. The time correlation functions and the power spectra provided a set of important information which allowed us to distinguish the two types of coexistence. Moreover, we have found that 
the power spectra present a tail, for high values of the frequency with slope equal to 2, characterizing the fluctuations in this regime as Brownian noise. Finally, the present analysis was used to examine real data.

\section{ACKNOWLEDGMENTS}

The authors have been supported by the Brazilian financial agencies CNPq and FAPESP.
[1] A. Lotka, Elements of Physical Biology (Williams and Wilkins, Baltimore, 1925).

[2] V. Volterra, Leçons sur la Théorie Mathématique de la Lutte pour la Vie (Gauthier-Villars, Paris, 1931).

[3] G. Nicolis and I. Prigogine, Self-Organization in Nonequilibrium Systems (Wiley, New York, 1977).

[4] E. Renshaw, Modelling Biological Populations in Space and Time (Cambridge University Press, Cambridge, 1991).

[5] A. Hastings, Population Biology: Concepts and Models (Springer, New York, 1997).

[6] M. Begon, C. R. Townsend, and J. L. Harper, Ecology, From Individuals to Ecosystems, 4th ed. (Blackwell, Malden, 2006).

[7] K. I. Tainaka, Phys. Rev. Lett. 63, 2688 (1989).

[8] J. E. Satulovsky and T. Tomé, Phys. Rev. E 49, 5073 (1994).

[9] R. Durrett and S. Levin, Theor. Popul. Biol. 46, 363 (1994).

[10] N. Boccara, O. Roblin, and M. Roger, Phys. Rev. E 50, 4531 (1994).

[11] J. Satulovsky and T. Tomé, J. Math. Biol. 35, 344 (1997).

[12] L. Frachebourg and P. Krapvisky, J. Phys. A 31, L287 (1998).

[13] A. Provata, G. Nicolis, and F. Baras, J. Chem. Phys. 110, 8361 (1999).

[14] A. Lipowski, Phys. Rev. E 60, 5179 (1999).

[15] R. Durrett and S. Levin, J. Theor. Biol. 205, 201 (2000).

[16] T. Antal, M. Droz, A. Lipowski, and G. Odor, Phys. Rev. E 64, 036118 (2001).

[17] T. Antal and M. Droz, Phys. Rev. E 63, 056119 (2001).

[18] M. A. M. Aguiar, H. Sayama, M. Baranger, and Y. Bar-Yam, Braz. J. Phys. 33, 514 (2003).

[19] K. C. de Carvalho and T. Tomé, Mod. Phys. Lett. B 18, 873 (2004).

[20] N. Nakagiri and K. Tainaka, Ecol. Modell. 174, 103 (2004).

[21] G. A. Tsekouras, A. Provata, and C. Tsallis, Phys. Rev. E 69, 016120 (2004).

[22] G. Szabó, J. Phys. A 38, 6689 (2005).

[23] D. Stauffer, A. Kunwar, and D. Chowdhury, Physica A 352, 202 (2005)

[24] C. Hauert and G. Szabó, Am. J. Phys. 73, 405 (2005).

[25] K. C. de Carvalho and T. Tomé, Int. J. Mod. Phys. C 17, 1647 (2006).

[26] S. Morita and K. Tainaka, Popul. Ecol. 48, 99 (2006).
[27] M. Mobilia, I. T. Georgiev, and U. C. Tauber, Phys. Rev. E 73, 040903(R) (2006).

[28] M. Mobilia, I. T. Georgiev, and U. C. Täuber, J. Stat. Phys. 128, 447 (2007)

[29] E. Arashiro and T. Tomé, J. Phys. A 40, 887 (2007).

[30] T. Tomé and K. C. de Carvalho, J. Phys. A 40, 12901 (2007).

[31] A. L. Rodrigues and T. Tomé, Braz. J. Phys. 38, 87 (2008).

[32] T. M. Liggett, Interacting Particle Systems (Springer, New York, 1985).

[33] J. Marro and R. Dickman, Nonequilibrium Phase Transitions in Lattice Models (Cambridge University Press, Cambridge, 1999).

[34] Metapopulation Biology: Ecology, Genetic and Evolution, edited by I. Hanski and M. E. Gilpin (Academic, San Diego, 1997).

[35] D. Tilman and P. Kareiva, Spatial Ecology: The Role of Space in Population Dynamics and Interactions (Princeton University Press, Princeton, NJ, 1997).

[36] A. Hastings, Theor. Popul. Biol. 12, 37 (1977).

[37] O. Ovaskainen, K. Sato, J. Bascompte, and I. Hanski, J. Theor. Biol. 215, 95 (2002).

[38] L. Berec, Ecol. Modell. 150, 55 (2002).

[39] W. W. Murdoch and E. McCauley, Nature (London) 316, 628 (1985).

[40] H. N. Comins, M. P. Hassell, and R. M. May, J. Anim. Ecol. 61, 735 (1992).

[41] W. G. Wilson, A. M. de Roos, and E. McCauley, Theor. Popul. Biol. 43, 91 (1993).

[42] E. McCauley, W. G. Wilson, and A. M. de Roos, Am. Nat. 142, 412 (1993).

[43] J. Bascompte, R. V. Solé, and N. Martinez, J. Theor. Biol. 187, 213 (1997).

[44] W. W. Murdoch, R. B. Nisbet, E. McCauley, A. M. de Roos, and W. S. C. Gurney, Ecology 79, 1339 (1998).

[45] X. Cai-lin and L. Zi-Zhen, J. Theor. Biol. 219, 73 (2002).

[46] S. Utida, Ecology 38, 442 (1957).

[47] C. J. Krebs, R. Boonstra, S. Boutin, and A. R. Sinclair, Bioscience 51, 25 (2001).

[48] N. C. Stenseth, W. Falck, O. N. Bjornstad, and C. J. Krebs, Proc. Natl. Acad. Sci. U.S.A. 94, 5147 (1997). 